\title{
Controle de Caos no Conversor Buck por meio do Método Pyragas
}

\author{
Adriano Rodrigues Neto ${ }^{1}$ \\ Departamento de Engenharia Elétrica, CEFET-MG, Belo Horizonte, MG \\ Everthon de Souza Oliveira ${ }^{2}$ \\ Departamento de Engenharia Elétrica, CEFET-MG, Belo Horizonte, MG \\ Programa de Pós-Graduação em Engenharia Elétrica - UFMG, Belo Horioznte, MG
}

\begin{abstract}
Resumo. Este artigo aborda o comportamento caótico do conversor Buck e apresenta uma metodologia de controle para tal condição. Esta metodologia se baseia no método Pyragas para controle de caos. O método foi capaz de controlar o caos aumentando a região de operação do conversor e melhorando a resposta na saída.
\end{abstract}

Palavras-chave. Conversor Buck, Caos, Controle, Método Pyragas, Não Linear

\section{Introdução}

A teoria do caos tem ganhado notoriedade nas últimas décadas, é apresentada inicialmente nos trabalhos de Edward Lorenz, em 1962, quando este estudava um modelo determinístico para fluxos não periódicos utilizando equações diferenciais. Ali foi visto que, condições iniciais ligeiramente diferentes poderiam desencadear em situações posteriores consideravelmente diferentes [8].

O comportamento caótico pode ser observado em diversas áreas das ciências como biologia, química, física ou economia por exemplo [7]. Existem estudos e técnicas que procuram construir um modelo de controle para que o sistema quando operando em regime caótico, responda de acordo com o regime nominal de operação, sem caos. O método Pyragas para controle de caos foi proposto em 1992 por K. Pyragas, em seus estudos envolvendo sistemas não lineares, este método propõe o controle do caos via realimentação por atraso estendido, o método é baseado na constante utilização de um laço de realimentação contendo informações de diversos estados anteriores do sistema [9]. Este método possui como vantagens de sua utilização, a robustez contra ruídos e a não necessidade de obter algum conhecimento analítico da dinâmica do sistema, além disto, a técnica pode ser utilizada de maneira relativamente simples.

O conversor buck é um ciruito que possui a capacidade de abaixar a tensão de saída para níveis menores que a tensão de entrada de acordo com a necessidade de projeto,

${ }^{1}$ rodrigues.a.neto@gmail.com

2 oliveira@deii.cefetmg.br 
conhecido também como conversor "step-down". Esta topologia é constituída na maioria ${ }^{3}$ das vezes de um indutor e duas chaves que geralmente são, um transistor e um diodo. Do ponto de vista básico de funcionamento, a energia flui entre a fonte de tensão e o indutor, para em um segundo momento, após o indutor carregado, esta energia seja fornecida à carga. Quando a chave mostrada na Figura 1 fecha, a tensão sob o indutor é $\mathrm{VL}=\mathrm{Voi}-\mathrm{V} 0$ e a corrente que ali passa linearmente aumenta. $\mathrm{O}$ diodo não permite a passagem da corrente por ele, uma vez que está polarizado invertidamente com relação a fonte. Quando a chave é aberta então, o diodo está polarizado diretamente e permite a passagem da corrente, a tensão no indutor será VL $=-\mathrm{V} 0$ e decrescerá. $\mathrm{O}$ esboço da situação é mostrado na Figura 1 abaixo [1].

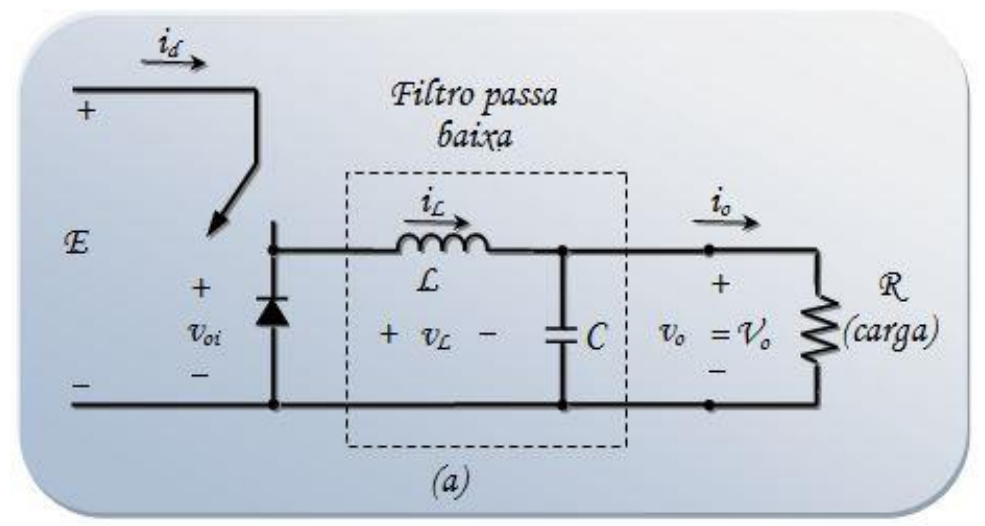

Figura 1: Esquemático conversor buck.

Tomando como base a Figura 1, utilizando-se as leis de Kirchhoff [1], é possível obter o modelo dinâmico do sistema composto por equações diferenciais. As equações são mostradas abaixo em função da razão cíclica da chave, sob o fator u.

$$
\begin{gathered}
\mathrm{L} \dot{\mathrm{X}}=-\boldsymbol{v}+\boldsymbol{u} \boldsymbol{E}(1) \\
\mathrm{C} \dot{\mathrm{y}}=\boldsymbol{i}-\frac{\boldsymbol{v}}{\boldsymbol{R}}(2)
\end{gathered}
$$

Este sistema de equações foi utilizado para realizar a simulação do modelo.

\section{Propósito}

Este trabalho tem como proposta inicial um estudo sobre a aplicação do método Pyragas para controle de caos em sistemas não lineares. O objetivo é utilizar esta ferramenta no controle do conversor de tensão Buck, aumentando assim, sua faixa de operação.

\section{Métodos}

\subsection{Método Pyragas}

Para a estabilização do sistema, foi utilizado o método Pyragas para controle de caos. Neste método, o controlador proposto para estabilizar uma órbita $\tau$-periódica de um sistema não linear possui a seguinte forma: 


$$
\boldsymbol{u}(\boldsymbol{t})=\boldsymbol{K}[\boldsymbol{x}(\boldsymbol{t})-\boldsymbol{x}(\boldsymbol{t}-\boldsymbol{\tau})(3)
$$

Sendo K o ganho da realimentação, e $\tau$ o tempo de atraso. Se $\tau$ for igual ao período de uma solução periódica existente de

$$
\dot{\mathrm{x}}=\boldsymbol{F}(\boldsymbol{u}, \boldsymbol{x})(4)
$$

para $\boldsymbol{u}=\mathbf{0}$, a solução $\boldsymbol{x}(\boldsymbol{t})$ da malha fechada do sistema (2) e (1) começa por $\Gamma=$ $\{\boldsymbol{x}(\boldsymbol{t})\}$, então restará um $\Gamma$ para todo $t \geq \mathbf{0}$ maior ou igual a 0 . Uma versão estendida do método de Pyragas também é proposta, como sendo:

$$
\boldsymbol{u}(\boldsymbol{t})=K \sum_{K=0}^{M} r k[y(t-k \tau)-y(t-(k+1) \tau)](5)
$$

Onde $\boldsymbol{y}=\boldsymbol{h}(\boldsymbol{x}(\boldsymbol{t}))$ pertence a R1 é a saída medida e rk, $\mathrm{k}=0, \ldots$, M são parâmetros de ajuste. Para $\mathrm{rk}=\mathrm{rk},|\mathrm{r}|<1$ e $\mathrm{M} \rightarrow \infty$ a lei do controle (3) se torna:

$$
\boldsymbol{u}(\boldsymbol{t})=\boldsymbol{K}[\boldsymbol{y}(\boldsymbol{t})-\boldsymbol{y}(\boldsymbol{t}-\boldsymbol{\tau})]+\boldsymbol{K r u}(\boldsymbol{t}-\boldsymbol{\tau})(6)
$$

Uma condição simples é necessária para a estabilização do controlador de Pyragas (1), o número de autovalores reais presente na matriz do modelo do sistema linearizado, perto do ponto fixo desejado, $\left(\frac{d F}{d x}(\mathrm{x}(0))\right)$, que são maiores que 1 não podem ser ímpar. Para um sistema escalar discreto $\mathrm{xk}+1=\mathrm{P}(\mathrm{xk}, \mathrm{uk})$ a condição necessária para a existência de uma versão discreta da realimentação de estabilização é $\lambda=\frac{d P}{d y}(\mathbf{0}, \mathbf{0})<1$. Ainda, o controlador de Pyragas modificado sugere que

$$
\boldsymbol{u}(\boldsymbol{t})=\boldsymbol{G}(\boldsymbol{p})[\boldsymbol{y}(\boldsymbol{t})-\boldsymbol{y}(\boldsymbol{t}-\boldsymbol{\tau})](7)
$$

Onde $\mathrm{G}(\mathrm{p}), \mathrm{p}=\frac{d}{d t}$ é a função de transferência do filtro e dá a condição de domínio da frequiência de estabilidade para o sistema de Lorenz. Usando a teoria absoluta da estabilidade, condições suficientes da função de transferência da parte linear do sistema controlado e a inclinação da não linearidade é obtida, sob a qual existe a estabilização $\mathrm{G}(\mathrm{p})[9]$.

\section{Resultados}

O conversaor de tensão possui um comportamento caótico quando seu funcionamento é submetido a alguns parâmetros de entrada [8]. Este comportamento é caracterizado pelo atrator estranho característico desta condição de funcionamento e mostrado na Figura 2. Este atrator é caracterizado pela tensão no capacitor e pela corrente na carga, quando plotados um pelo outro. 


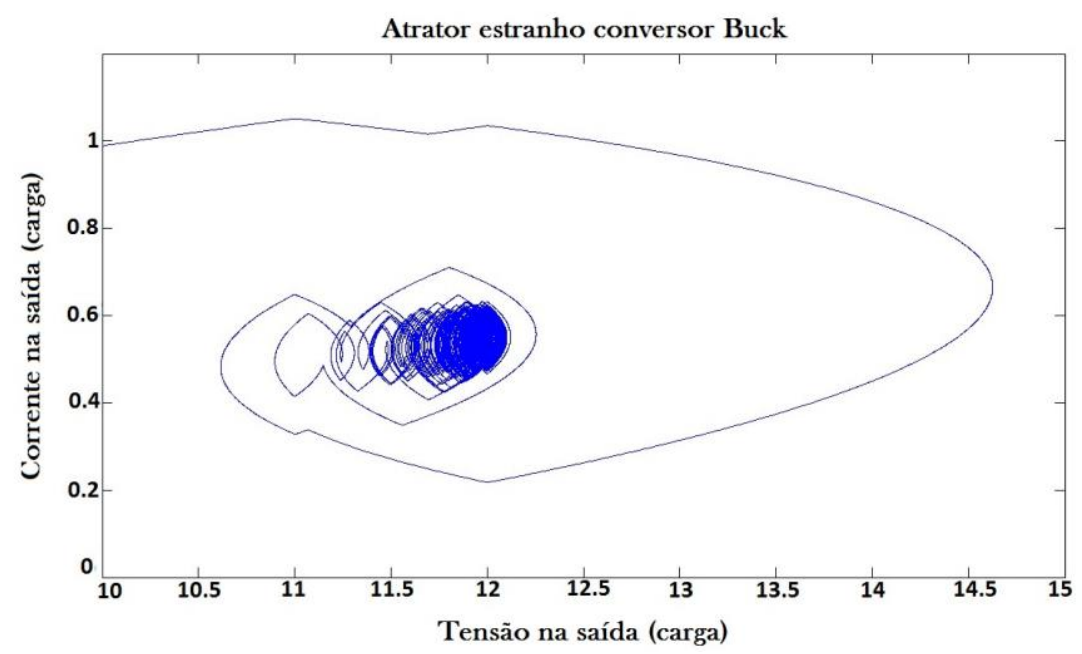

Figura 2: Forma do Atrator Estranho. Vref $=11.3 \mathrm{~V}, \mathrm{R}=22 \Omega, \mathrm{L}=20 \mathrm{mH}, \mathrm{C}=47 \mathrm{mF}$, $\mathrm{f}($ chave $)=2.5 \mathrm{kHz}[2]$

O diagrama de bifurcação do conversor também é utlizado para um parâmetro característico desta condição, neste diagrama, é possível perceber para quais regiões de dado parâmetro variável a condição caótica é presente ou não. O diagrama de bifuração mostrado na Figura 3 é visto em [4]. É possível perceber que a tensão de entrada foi variada em função da corrente do indutor.

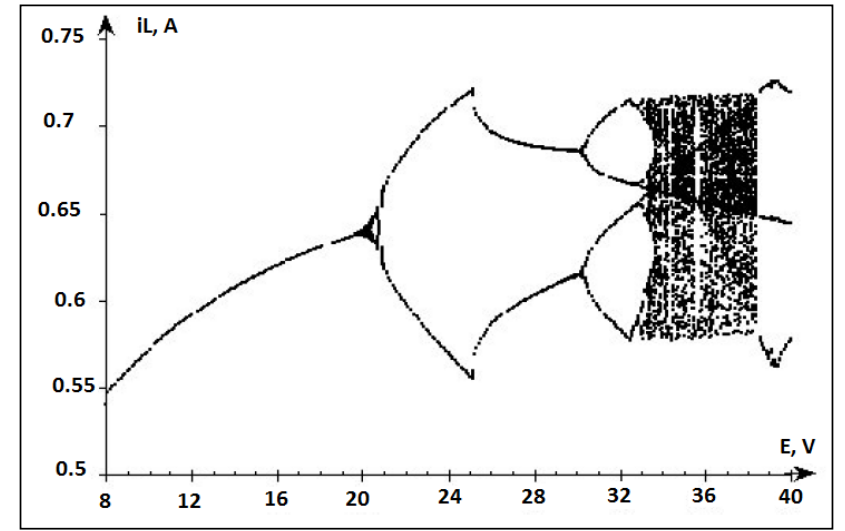

Figura 3: Diagrama de bifurcação [4]

Ainda, é possível perceber um comportamento análogo na tensão de saída para os mesmos parâmetros de simulação, situação mostrada na figura 4 . 


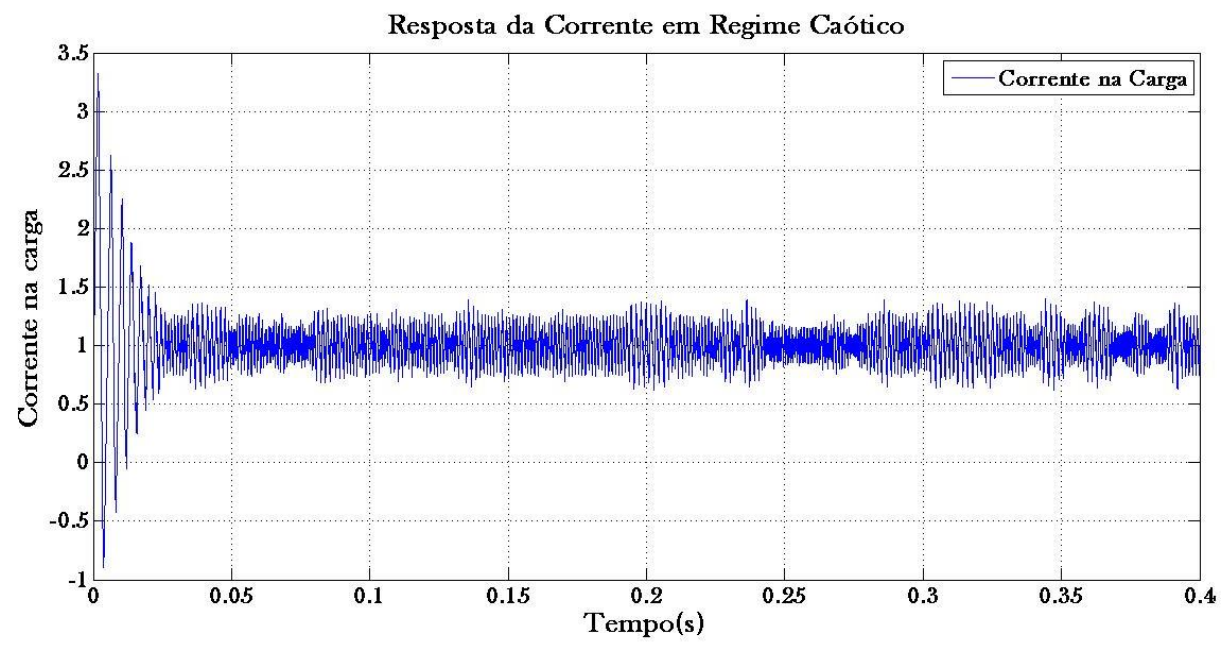

Figura 4: Resposta em regime caótico da corrente na saída

O circuito controlado pelo método de Pyragas apresenta as formas de onda da corrente e tensão de saída na carga como visto na Figura 5 e Figura 6, respectivamente.

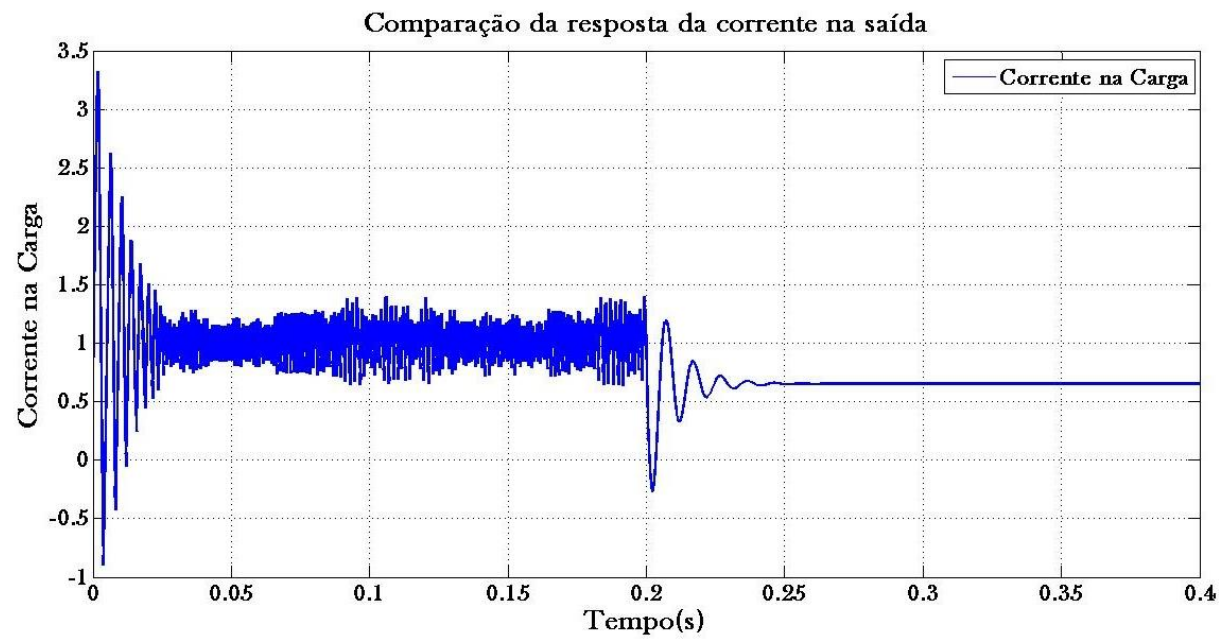

Figura 5: Forma de onda da corrente na carga, antes e após a ação de controle 


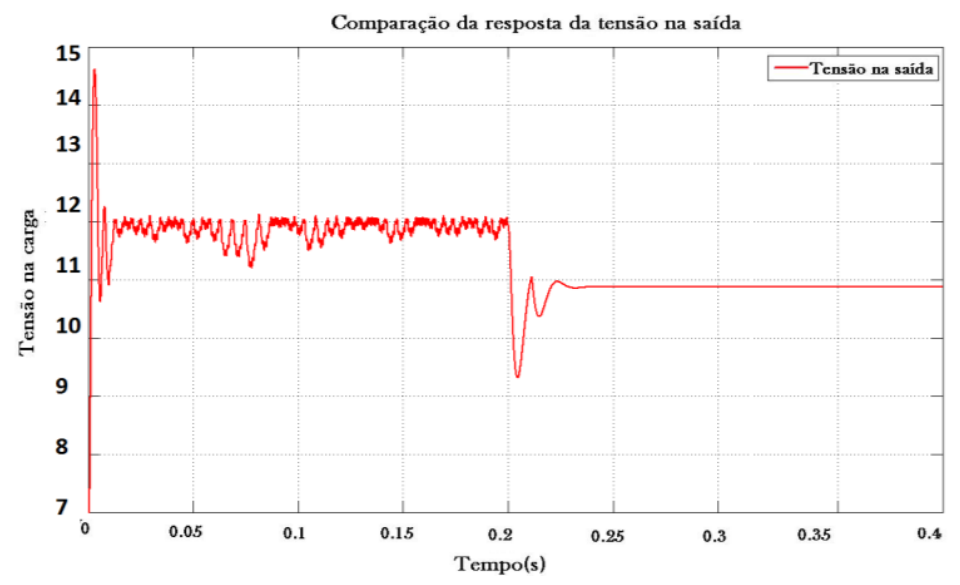

Figura 6: Forma de onda da tensão de saída, antes e após a ação de controle

\section{Discussões e conclusões}

É possível perceber a eficácia do método Pyragas para controle de caos quando aplicado ao conversor de tensão buck em regime caótico. Na Figura 5 e na Figura 6 existe dois períodos diferentes de operação. Estes dois períodos são caracterizados pela transição da resposta do sistema a partir da ação de uma chave ideal, este elemento é capaz de comutar a ação controladora e impor tal condição ao sistema. Sendo assim, percebe-se que antes de $0.2 \mathrm{~s}$ o sistema se encontra em regime caótico e de $0.2 \mathrm{~s}$ até $0.4 \mathrm{~s}$, o sistema é mostrado já com a ação do controle.

Após a aplicação do método, tanto a tensão na saída quanto a corrente no indutor são estabilizados, isto é visto na Figura 5 e na Figura 6. O controle Pyragas além de estabilizar a saída, leva a tensão para um valor mais próximo da referência esperada. É importante ressaltar também a simplicidade do método e sua aplicação.

\section{Referências}

[1] C. Batlle, E. Fossas and G. Olivar, Time-delay stabilization of the buck converter, Proc. of the 1st International Conf. on Control of Oscillations and Chaos, (1997).

[2] N. K. Chiang, N. T. M. Lin and M. T. T. Tien, Bifurcation Behaviour of the Buck Converter, Journal - The Institution of Engineers, Vol. 75, (2014).

[3] Converter System Modeling via MATLAB/Simulink, Department of Electrical, Computer, and Energy Engineering.

[4] I. Flegar, D. Pelling and D Zacek, Bifurcation Diagrams of the buck converter.

[5] J. Forsyth and S. V. Mollov, Modeling and Control of DC-DC converters, Power Engineering Journal, (1998). 
[6] Introduction to MATLABA/Simulink for switched mode power converters, Colorado Power Electronics Center.

[7] S. Iqbal, M. Ahmed, and S. A. Qureshi, Investigation of Chaotic Behavior in DC-DC Converters, International Journal of Electrical, Computer, Electronics and Communication Engineering Vol. 1, (2007).

[8] E. N. Lorenz, Deterministic Nonperiodic Flow, Journal of the Atmospheric Sciences, Vol. 20,

[9] K. Pyragas, Continuous control of chaos by self-controlling feedback, Elsevier SciencePublishers B.V., (1992).

[10] H. Sira-Ramirez and R. Silva-Ortigoza, Control Design Techniques in Power Electronics Devices, Chapter 2, Vol. 1, (2006), DOI: 10.1007/1-84628-459-7.

[11] E. R. Vilamitjana, A. El Aroudi, and E. Alarcón, Chaos in Switching Converters for Power Management: Designing for Prediction and Control, Chapter 2, Vol. 1, (2013), DOI: 10.1007/978-1-4614-2128-3. 